\title{
Circulating fatty acid synthase: an exploratory biomarker to predict efficacy of the dual HER1/ HER2 tyrosine kinase inhibitor lapatinib
}

\author{
Cristina Oliveras-Ferraros, ${ }^{1,2}$, Sílvia Cufi',2, Tamara Sauri-Nadal 2,3, Sonia Del Barco ${ }^{2,3}$, Begoña Martin-Castillo, \\ Alejandro Vazquez-Martin ${ }^{1,2}$ and Javier A Menendez ${ }^{* 1,2}$ \\ See related research by Jin et al., http://breast-cancer-research.com/content/12/6/R96
}

In the previous issue of Breast Cancer Research, Jin and colleagues [1] demonstrated that the lipogenic enzyme fatty acid synthase (FASN) is phosphorylated when it is in complex with the tyrosine kinase receptor HER2. HER2-dependent activating phosphorylation of FASN was related to tumor progression and was sensitive to the dual HER1/HER2 tyrosine kinase inhibitor lapatinib [1]. Adding to knowledge of the therapeutic value of the phosphorylation status of FASN in HER2-driven breast cancer (BC), we now provide experimental evidence that the expression status of FASN in the extracellular milieu (that is, extracellular FASN) may function as a novel biomarker that distinctively predicts molecular functioning of lapatinib.

AMP-activated protein kinase (AMPK)-activating drugs, by mimicking an elevated AMP/ATP ratio in BC cells, drastically augment the release of extracellular FASN in HER2-positive BC cells [2]. Lapatinib-induced deprivation of tumor cell energy activates AMPK to trigger an entire cascade of metabolic events, including suppression of FASN expression and activity $[1,3]$. We hypothesized that a differential ability to initiate AMPKsensed metabolic stress responses may provide information about the efficacy of HER-targeting drugs via changes in the extracellular FASN status. Enzyme-linked immunosorbent assay (ELISA)-based quantitative analyses revealed that lapatinib treatment drastically enhanced extracellular FASN concentration (by at least 8.0-fold) (Figure 1a). Immunoblotting assessment of AMPK phosphorylation at Thr172 confirmed that lapatinib treatment induced a strong activation of AMPK

\footnotetext{
*Correspondence: jmenendez@iconcologia.net

'Unit of Translational Research, Catalan Institute of Oncology-Girona (ICO-Girona),

1-4, Ctra. França s/n, E-17007 Girona, Catalonia, Spain

Full list of author information is available at the end of the article
}

(Figure 1b). A weak but detectable upregulation of PPAMPK $^{\text {Thr172 }}$ was observed upon treatment with gefitinib. Trastuzumab, cetuximab, and erlotinib - all of which are unable to promote FASN release - failed to activate AMPK. AMPK knockdown using short interfering RNA (siRNA) transfection [2] fully prevented lapatinibinduced FASN release (Figure S1). Immunoblotting and cell imaging analyses confirmed that FASN was depleted from the cytosol of lapatinib-treated HER2 overexpressors and accumulated in their extracellular milieu (Figure S1).

Treatment with lapatinib dramatically increased FASN release (by approximately 17 times) in lapatinib-responsive SKBR3 TzbR cells (Figure 1c), which were selected for long-term outgrowth in trastuzumab-containing culture medium. Extracellular FASN remained unaltered in response to trastuzumab or lapatinib in JIMT-1 BC cells, which exhibit de novo cross-refractoriness to multiple HER1/2-targeted therapies (Figure 1c). Lapatinib treatment significantly activated AMPK and promoted an enormous accumulation of extracellular FASN in lapatinib-hypersensitive MCF-7/HER2 cells (Figure 1d). Equimolar concentrations of lapatinib failed to activate AMPK or to alter FASN release into the extracellular milieu of MCF-7/HER2 LapR cells, which were obtained by continuously subculturing parental MCF-7/HER2 cells with high-dose lapatinib.

High levels of circulating FASN can be found in peripheral blood of HER2-overexpressing BC patients [4]. The exclusive ability of lapatinib to actively promote the extracellular release of FASN via AMPK-sensed energy depletion in metabolically demanding HER2driven BC cell growth might provide a molecular rationale to evaluate the predictive value of circulating FASN in HER2-positive BC patients receiving lapatinib.

\section{Abbreviations}

AMPK, AMP-activated protein kinase; BC, breast cancer; FASN, fatty acid synthase. 


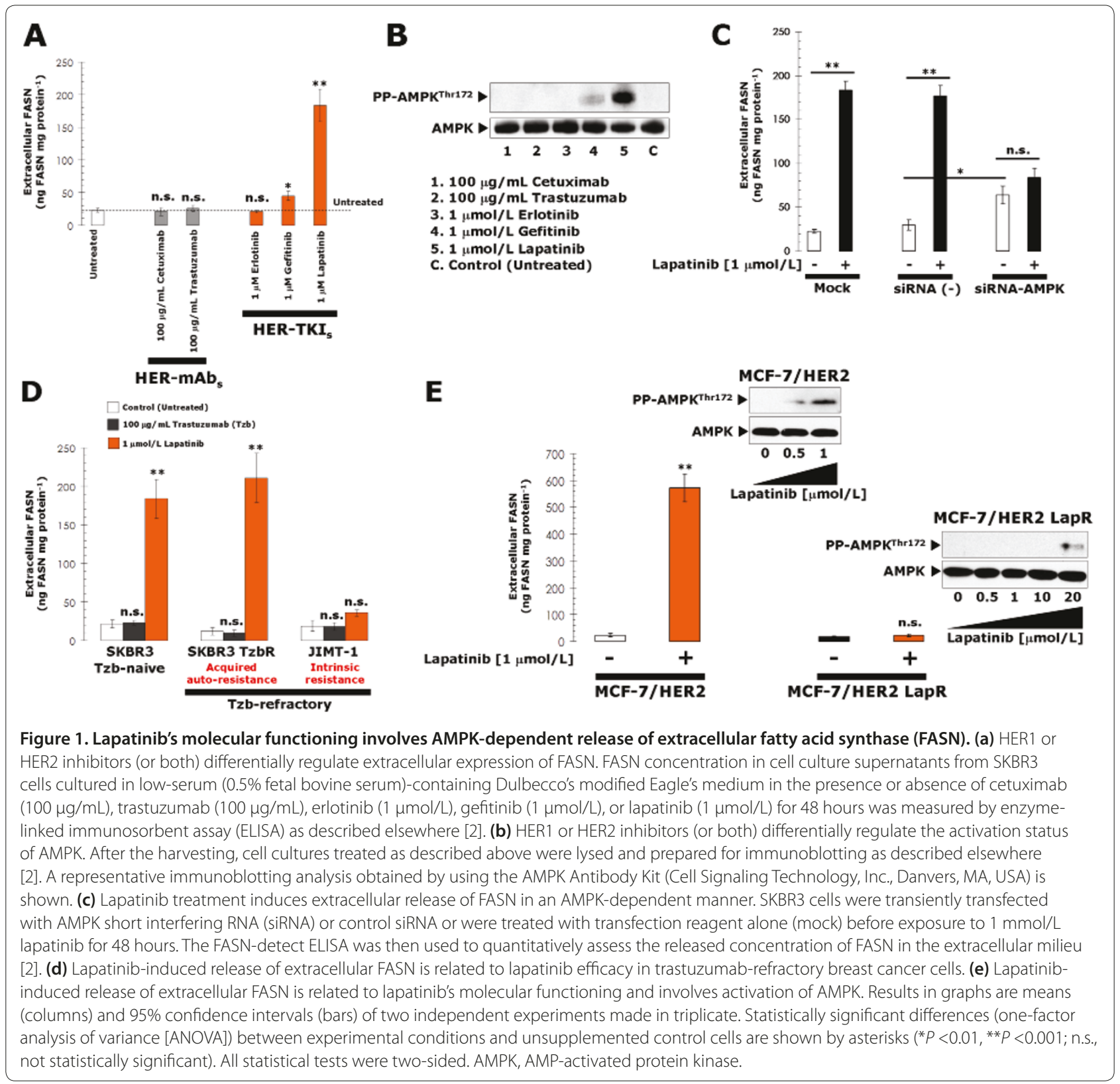

\section{Competing interests}

The authors declare that they have no competing interests.

\section{Acknowledgments}

The authors wish to dedicate this letter to the memory of Dr. Francis $P$ Kuhajda, who passed away suddenly 10 November 2010. Dr. Kuhajda was an associate professor of pathology, oncology, and biological chemistry at Johns Hopkins University (JHU) (Baltimore, MD, USA) and director of the JHU Center for Metabolic Pathology. He pioneered the exploration of basic molecular mechanisms linking cancer cell apoptosis with inhibition of fatty acid synthase (FASN)-catalyzed endogenous fatty acid biogenesis. He was actively involved in translational research developing new inhibitors of FASN for future clinical use. He discovered that FASN, a cytosolic protein, can also be found circulating in the blood of patients with cancer. Delineating the forms of the circulating FASN antigen and understanding its role in cancer diagnosis and prognosis were among his ongoing projects.
SC is the recipient of a research fellowship (Formación de Personal Investigador, or FPI) by the Ministerio de Ciencia e Innovación (MICINN, Spain). AV-M is the recipient of a 'Sara Borrell' post-doctoral contract (CD08/00283, Ministerio de Sanidad y Consumo, Fondo de Investigación Sanitaria [FIS], Spain). Work at the laboratory of JAM is supported in part by the Instituto de Salud Carlos III (Ministerio de Sanidad y Consumo, FIS, Spain, grants CP05-00090, PI06-0778, and RD06-0020-0028), the Fundación Científica de la Asociación Española Contra el Cáncer (AECC, Spain), and the Ministerio de Ciencia e Innovación (SAF2009-11579, Plan Nacional de I+D+ I, MICINN, Spain).

\section{Author details}

'Unit of Translational Research, Catalan Institute of Oncology-Girona (ICOGirona), 1-4, Ctra. França s/n, E-17007 Girona, Catalonia, Spain; ${ }^{2}$ Girona Biomedical Research Institute (IdIBGi), 1-4, Ctra. França s/n, E-17007 Girona, Catalonia, Spain; ${ }^{3}$ Medical Oncology, Catalan Institute of Oncology-Girona (ICO-Girona), 1-4, Ctra. França s/n, E-17007 Girona, Catalonia, Spain; ${ }^{4} U n i t$ of 
Clinical Research, Catalan Institute of Oncology-Girona (ICO-Girona), 1-4, Ctra. França s/n, E-17007 Girona, Catalonia, Spain.

\section{Supplementary material}

Figure S1. Lapatinib treatment promotes the release of two extracellular forms of fatty acid synthase (FASN). Left: Extracellular expression of FASN in SKBR3 cultures exposed to serum starvation (lower panels) or 1 umol/L lapatinib (upper panels) for 0, 6, 24, and 48 hours was monitored by immunoblotting procedures using three monoclonal anti-FASN antibodies (Abs) as specified [2]. FASN1 indicates the high-molecular-mass (260 kDa) form of FASN, and FASN2 indicates the intermediate-size $(150 \mathrm{kDa})$ form of FASN. The clone M3 (1:2,000 dilution), a mouse anti-FASN monoclonal antibody (kindly provided by Ellen Pizer, Johns Hopkins University, Baltimore, MD, USA) diluted 1:5,000; the clone 23 (1:250 dilution), a mouse anti-FASN monoclonal antibody obtained from BD Biosciences Pharmingen (San Diego, CA, USA); and the clone 3B3-1D6 (1:250 dilution), a mouse anti-FASN monoclonal antibody (M02) obtained from Abnova (Taipei, Taiwan), were used. Right: Images of FASN expression in whole SKBR3 cell cultures before and after treatment with $1 \mu \mathrm{mol} / \mathrm{L}$ lapatinib were captured as a 4-by-4 montage on a BD Pathway ${ }^{\mathrm{TM}} 855$ Biolmager System (BD Biosciences Pharmingen). Two different channels for Alexa Fluor 488 (pseudo-colored red: FASN) and Hoechst 33342 (pseudo-colored blue: nuclei) were used. Images were captured with a 20x objective (NA 075; Olympus, Tokyo, Japan) in accordance with the recommended assay procedure and were merged by means of $\mathrm{BD}$ Attovision ${ }^{\mathrm{TM}}$ software (BD Biosciences Pharmingen). A schematic depicting a model of the relationship between lapatinib-responsive, AMP-activated protein kinase (AMPK)sensed cellular energy status and extracellular release of FASN in HER2-positive breast cancer cells is shown.
Published: 24 January 2011

\section{References}

1. Jin Q, Yuan LXH, Boulbes DR, Baek J-M, Wang Y-N, Gomez-Cabello D, Hawke DH, Yeung S-C, Lee M-H, Hortobagyi GN, Hung M-C, Esteva FJ: Fatty acid synthase phosphorylation: a novel therapeutic target in HER2overexpressing breast cancer cells. Breast Cancer Res 2010, 12:R96.

2. Oliveras-Ferraros C, Vazquez-Martin A, Fernández-Real JM, Menendez JA: AMPK-sensed cellular energy state regulates the release of extracellular fatty acid synthase. Biochem Biophys Res Commun 2009, 378:488-493.

3. Shell SA, Lyass L, Trusk PB, Pry KJ, Wappel RL, Bacus SS: Activation of AMPK is necessary for killing cancer cells and sparing cardiac cells. Cell Cycle 2008, 7:1769-1775.

4. Vazquez-Martin A, Fernandez-Real JM, Oliveras-Ferraros C, Navarrete JM, Martin-Castillo B, Del Barco S, Brunet J, Menendez JA: Fatty acid synthase activity regulates HER2 extracellular domain shedding into the circulation of HER2-positive metastatic breast cancer patients. Int J Oncol 2009, 35:1369-1376. 